\title{
Numerical analysis of a highly birefringent microstructured optical fiber with an anisotropic core
}

\author{
Michal Swat • Bartlomiej Salski · Tomasz Karpisz • \\ Grzegorz Stepniewski • Ireneusz Kujawa • \\ Mariusz Klimczak • Ryszard Buczynski
}

Received: 12 January 2014 / Accepted: 4 July 2014 / Published online: 17 July 2014

(C) The Author(s) 2014. This article is published with open access at Springerlink.com

\begin{abstract}
In this paper, modeling and optimization of a highly birefringent microstructured optical fiber with an anisotropic structure of a lamellar core is analyzed. The core consists of a linear stack of a high refractive index lead oxide glass F2 and a low refractive index borosilicate glass NC21A, which contributes to the anisotropy of two orthogonal polarizations of the fundamental mode propagating in the fiber. It is shown, that an appropriate choice of thickness and width of the layers constituting the core structure, enables reducing the dispersion of birefringence of the considered modes, in a wide spectral range. It is further investigated how a sub-wavelength defect, in form of a low refractive index glass introduced in the middle of the core, influences fiber's birefringence. We show for the first time, that nanodefect introduced into a lamellar core structure further reduces dispersion of birefringence in the fiber over one octave range. An average birefringence of $1.95 \times 10^{-3}$ with variation below $5 \%$ is achieved in $800-2,000 \mathrm{~nm}$ bandwidth.
\end{abstract}

Keywords Highly birefringent fiber · Microstructure fibers · Anisotropic materials · Finite difference time domain method

\section{Introduction}

Birefringence in optical fibers has been attracting scientific interest for over 30 years (Ulrich and Simon 1979). Various methods for introduction of bierfringence in optical fibers were

\footnotetext{
M. Swat · B. Salski · T. Karpisz

Institute of Radioelectronics, Warsaw University of Technology, Nowowiejska 15/19, 00-665 Warsaw, Poland
}

M. Swat · T. Karpisz · G. Stepniewski - I. Kujawa - M. Klimczak · R. Buczynski Institute of Electronic Materials Technology, ul. Wólczyńska 133, 01-919 Warsaw, Poland

G. Stepniewski · R. Buczynski ( $\square)$

Faculty of Physics, University of Warsaw, ul. Pasteura 7, 02-093 Warsaw, Poland

e-mail: rbuczyns@igf.fuw.edu.pl 
studied (Klein and Heinlein 1982; Varnham et al. 1983). Recently, highly birefringent fibers are one of the most successful applications of microstructured optical fibers (MOFs) (Ortigosa-Blanch et al. 2000). Due to flexibility of design, this approach offers various mechanisms to introduce two-fold symmetry in a fiber. MOFs usually exhibit much higher birefringence values than their conventional counter-parts such as elliptical core, bow tie and panda type optical fibers (Frazao et al. 2008). MOFs with air holes benefit from low temperature sensitivity, therefore they are good candidates for various mechanical sensors such as strain, stress or pressure (Martynkien et al. 2009). A lot of interest attract MOFs with elliptical holes in a hexagonal or rectangular lattice, since they offer theoretically very high birefringence in the order of $\mathrm{B}=10^{-2}$ (Yue et al. 2007). However, practical parameters of these types of structures are limited by available technology and their fabrication repeatability (Buczynski et al. 2010; Kujawa et al. 2012; Beltrán-Mejía et al. 2010).

Development of all-solid birefringent MOFs is studied as an alternative approach. Various two-fold geometries were tested for the development of highly birefringent photonic bandgap fibers (Goto et al. 2008) or single polarization fibers (Cerqueira et al. 2011; Goto et al. 2009). Another all-solid glass approach was proposed by Wang at al. (Wang et al. 2005), where birefringence was induced by effective anisotropy of subwavelength structure of the core, instead of commonly used birefringence of photonic cladding of the fibers. The considered fiber consisted of several interleaved layers built of two commercial Schott glasses: SF6 $\left(n_{d}=\right.$ $1.79)$ and LLF1 $\left(\mathrm{n}_{\mathrm{d}}=1.54\right)$. This type of core structure was originally named as 'lamellar core'. Similar approach was used by Waddie at al. for development of volumetric anisotropic materials (Waddie et al. 2011). However in this case, another set of lead-silicate glass F2 and silicate glass NC21A was used. According to performed simulations, an anisotropic structure shows similar birefringence of $2 \times 10^{-3}$ in a broad wavelength range of $800-2,000 \mathrm{~nm}$. In this paper, a complete electromagnetic (EM) design cycle and an optimization process of a highly birefringent fiber with an anisotropic lamellar core made of F2 and NC21A glasses is demonstrated.

Broadbandly flat birefringence in optical fibers is required in interferometric sensor systems, where broadband sources are used. It allows to keep similar sensitivity of measurement in all measurement range (Frazao et al. 2007). In this paper, for the first time to our best knowledge, we consider introduction of a nanodefect in the lamellar core and we study its influence on birefringence characteristics. Use of a nanostructure in the fiber core was successfully verified in case of development of nonlinear all-solid MOFs with flat dispersion (Buczynski et al. 2011). The study is undertaken with the aid of a finite difference time domain (FDTD) method (Taflove and Hagness 2005), implemented in the QuickWave software package (QuickWave-3D 1997).

\section{Electromagnetic model of birefringent fiber with a lamellar core}

In this section we investigate a MOF with an anisotropic lamellar core. The core is made of two types of glasses, commercial F2 $\left(\mathrm{n}_{\mathrm{d}}=1.619\right)$ and in-house synthesized NC21A $\left(\mathrm{n}_{\mathrm{d}}=1.518\right)$, stacked alternately, and surrounded with NC21A glass. We denote X axis along layers in the core and $\mathrm{Y}$ axis perpendicular to layers in the core. Details of both glasses were provided in (Lorenc et al. 2008). Since the geometry is invariant along the fiber, an FDTD analysis is limited to a transverse cross-section of the fiber with an analytically imposed longitudinal phase constant $\beta_{\mathrm{f}}$. Moreover, the symmetry of both the structure and the considered modes enables further reduction of a two-dimensional (2D) analysis to the quarter of the cross-section. As it is shown in Fig. 1, two boundaries of the FDTD model 


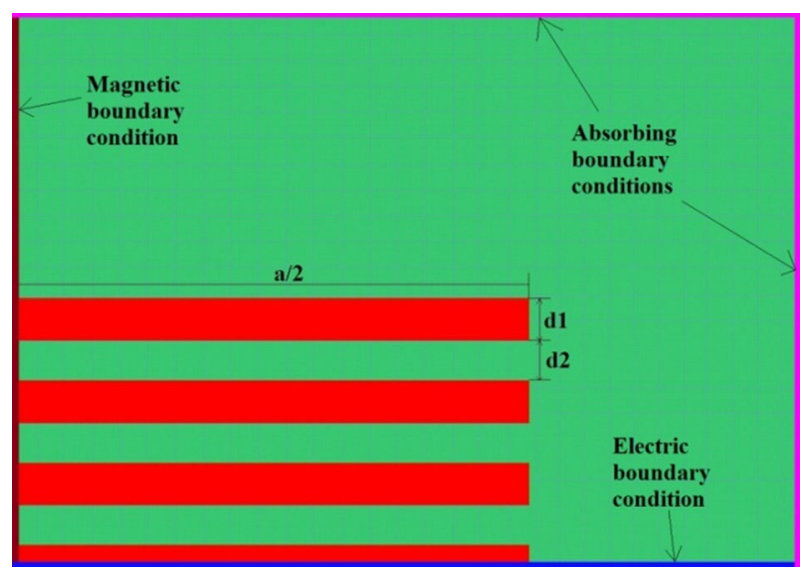

Fig. 1 An FDTD model of a quarter of a microstructured optical fiber's cross-section

are equipped with magnetic and electric boundary conditions, while the remaining sides are terminated with absorbing boundary conditions (Mei and Fana 1992). Consequently, computational effort is substantially reduced.

Refractive index $n$ for each of the glasses is given by the Sellmeier equation:

$$
\mathrm{n}^{2}(\lambda)=1+\frac{B_{1} \lambda^{2}}{\lambda^{2}-C_{1}}+\frac{B_{2} \lambda^{2}}{\lambda^{2}-C_{2}}+\frac{B_{3} \lambda^{2}}{\lambda^{2}-C_{3}}
$$

where $\lambda$ is a wavelength, and $B_{1,2,3}$ and $C_{1,2,3}$ are Sellmeier coefficients. The model is converted to a triple-pole Lorentz model, which is well-known in FDTD routines:

$$
\begin{aligned}
\varepsilon_{\mathrm{r}}(\omega)= & \varepsilon_{\infty}+\mathrm{A}_{1} \frac{\left(\varepsilon_{\mathrm{S}}-\varepsilon_{\infty}\right)\left(2 \pi \mathrm{f}_{\mathrm{p} 1}\right)^{2}}{\left(2 \pi \mathrm{f}_{\mathrm{p} 1}\right)^{2}+\mathrm{j} \omega 2 \pi \mathrm{f}_{\mathrm{C} 1}-\omega^{2}} \\
& +\mathrm{A}_{2} \frac{\left(\varepsilon_{\mathrm{S}}-\varepsilon_{\infty}\right)\left(2 \pi \mathrm{f}_{\mathrm{p} 2}\right)^{2}}{\left(2 \pi \mathrm{f}_{\mathrm{p} 2}\right)^{2}+\mathrm{j} \omega 2 \pi \mathrm{f}_{\mathrm{C} 2}-\omega^{2}}+\mathrm{A}_{3} \frac{\left(\varepsilon_{\mathrm{S}}-\varepsilon_{\infty}\right)\left(2 \pi \mathrm{f}_{\mathrm{p} 3}\right)^{2}}{\left(2 \pi \mathrm{f}_{\mathrm{p} 3}\right)^{2}+\mathrm{j} \omega 2 \pi \mathrm{f}_{\mathrm{C} 3}-\omega^{2}}
\end{aligned}
$$

where $\varepsilon_{\infty}$ is optical relative permittivity, $\varepsilon_{s}$ is static relative permittivity, $f_{p 1,2,3}$ stand for frequencies of poles, $f_{c 1,2,3}$ represent relaxation frequencies, and $A_{1,2,3}$ denote weight coefficients of the corresponding dispersive poles. Table 1 shows Sellmeier and Lorentz coefficients for both types of glasses.

Sellmeier coefficients are converted into Lorentz coefficient with following equations:

$$
\begin{aligned}
& A_{1}=1, \varepsilon_{S}=A_{1}\left(B_{1}-1\right), A_{2}=\frac{B_{2}}{\varepsilon S^{-1}}, A_{3}=\frac{B_{3}}{\varepsilon S^{-1}} \\
& f_{p 1}=\frac{c}{\sqrt{C_{1}}}, f_{p 2}=\frac{c}{\sqrt{C_{2}}}, f_{p 3}=\frac{c}{\sqrt{C_{3}}}
\end{aligned}
$$

To determine birefringence in a given spectral range, several FDTD simulations need to be performed for a given range of the longitudinal phase constant $\beta_{f}$ :

$$
\beta_{f}=\frac{2 \pi}{\lambda_{0}} n_{e f f}=\frac{2 \pi f}{c} n_{e f f}
$$

In each simulation iteration for the imposed $\beta_{f}$, the structure shown in Fig. 1 is excited with a point source driven with the Kronecker delta to cover the whole investigated spectrum. 
Table 1 Sellmeier and Lorentz coefficients of the lead-silicate F2 and silicate NC21A glasses

\begin{tabular}{llcc}
\hline Model type & Parameters & Glass type & \\
\cline { 3 - 4 } Sellmeier & & NC21A & $\mathrm{F} 2$ \\
\hline & $\mathrm{B}_{1}$ & 1.15702 & 1.34533 \\
& $\mathrm{~B}_{2}$ & 0.149600 & 0.20907 \\
& $\mathrm{~B}_{3}$ & 1.36007 & 0.93736 \\
& $\mathrm{C}_{1}\left(\mu \mathrm{m}^{2}\right)$ & 0.00614 & 0.009980 \\
& $\mathrm{C}_{2}\left(\mu \mathrm{m}^{2}\right)$ & 0.02522 & 0.04704 \\
& $\mathrm{C}_{3}\left(\mu \mathrm{m}^{2}\right)$ & 122.84413 & 111.88676 \\
& $\varepsilon_{\infty}$ & 1.00000 & 1.00000 \\
& $\varepsilon_{S}$ & 2.15700 & 2.34530 \\
& $\mathrm{f}_{p 1}(\mathrm{PHz})$ & 3.82556 & 3.00136 \\
& $\mathrm{f}_{p 2}(\mathrm{PHz})$ & 1.88786 & 1.38226 \\
& $\mathrm{f}_{p 3}(\mathrm{PHz})$ & 0.0270494 & 0.0283424 \\
$\mathrm{~A}_{1}$ & 1.00000 & 1.00000 \\
& $\mathrm{~A}_{2}$ & 0.12930 & 0.15540 \\
$\mathrm{~A}_{3}$ & 1.17550 & 0.69670 \\
\hline
\end{tabular}

The next step is identification of the fundamental mode and its frequency $f$. Those results, together with (3), allow determining effective refractive index $n_{e f f}$ of a mode for the imposed $\beta_{f}$. That procedure has to be performed for both vertical $\left(\mathrm{E}_{\mathrm{y}}\right)$ and horizontal $\left(\mathrm{E}_{\mathrm{x}}\right)$ polarization components of the mode, by exchanging electric and magnetic boundary conditions depicted in Fig. 1. Subsequently, birefringence $B$ can be obtained as:

$$
B=n_{\text {effX }}-n_{\text {eff } Y},
$$

where $n_{e f f_{X}}, n_{e f f_{Y}}$ are effective refractive indices of both fundamental modes. The time complexity of proposed algorithm is similar to other commonly used methods for modeling of MOFs as finite difference or finite element method. Most of the currently used methods for modeling of linear properties of MOFs use the same approach, where spectral plots of effective refractive index are obtained on a point by point basis. In some cases methods (Finite element method, finite difference) are adopted to trace a solution only in the proximity of a given mode (mode spectral tracing). In our case we obtain solutions for all the spectrum in parallel (Salski et al. 2010).

\section{Modeling results}

In this Section, the impact of the geometry of the fiber on the dispersion and birefringence is evaluated. First, a lattice constant $\Lambda$ is swept from $300 \mathrm{~nm}$ up to $900 \mathrm{~nm}$ :

$$
\Lambda=d_{1}+d_{2},
$$

while maintaining a filling factor:

$$
f=\frac{d_{1}}{d_{2}}
$$

equal to $f=1$, where $d_{1}, d_{2}$ stand for the thickness of the strips inside the lamellar core as depicted in Fig. 1. Table 2 presents the list of considered dimensions of the scenario, 
Table 2 Geometrical parameters of the tested fiber structures

\begin{tabular}{lrrr} 
Filling factor $f=1$ & & & \\
$\Lambda(\mathrm{nm})$ & 300 & 600 & 900 \\
$d 1(\mathrm{~nm})$ & 150 & 300 & 450 \\
$d 2(\mathrm{~nm})$ & 150 & 300 & 450 \\
$a(\mathrm{~nm})$ & 3,580 & 7,160 & 10,740 \\
\hline
\end{tabular}

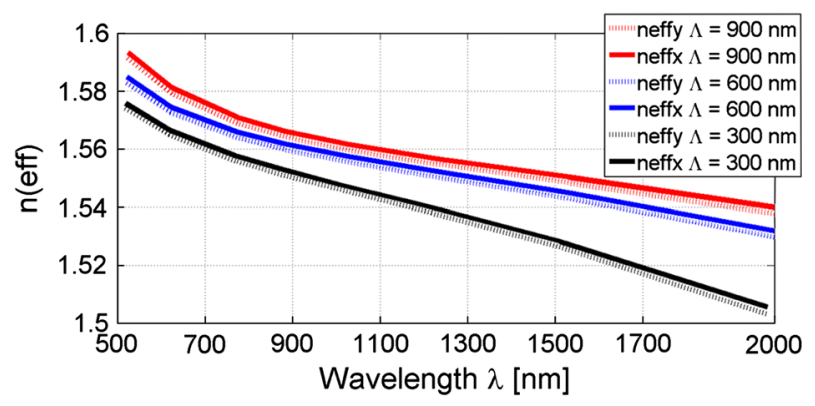

Fig. 2 Effective refractive index for the polarization component of fundamental mode $\left(E_{x}\right.$ and $\left.E_{y}\right)$ for various lattice constants $\Lambda$. The polarization component $E_{x}$ denotes direction of electric field vector along layers in the core (X axis), while $E_{y}$ denotes direction of electric field vector perpendicular to layers in the core ( $\mathrm{Y}$ axis)

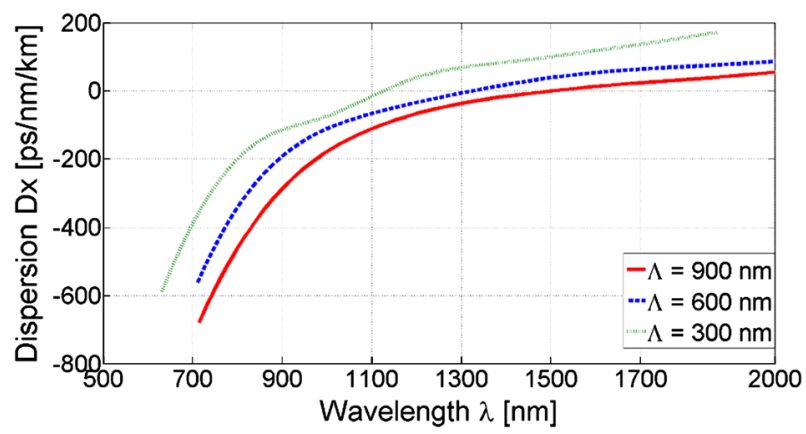

Fig. 3 Group velocity dispersion of the horizontally polarized component of fundamental mode $\left(E_{X}\right)$ for various lattice constants $\Lambda$. The polarization component $E_{\mathrm{X}}$ denotes direction of electric field vector along layers in the core (X axis)

indicating that the length $a$ of the strips is rescaled with the lattice constant $\Lambda$, accordingly. We assume that the fiber core consists of seven layer pairs of high and low refractive index glass. Therefore core is rectangular with diameters is $a \times b$, where $b=7 \Lambda$ and $a$ is given arbitrarily. However core aspect ratio $a / b$ is constant in all considered cases.

A series of EM simulations of the structure shown in Fig. 1, were performed using FDTD. Figures 2, 3 and 4 show obtained effective refractive index and dispersion profiles of vertically and horizontally polarized fundamental modes are shown, respectively. It can be seen that dispersion of both fundamental modes is very similar and can be controlled by adjustment of the lattice constant $\Lambda$. A zero dispersion wavelength (ZDW) increases with the increasing lattice constant $\Lambda$ from $1.2 \mu \mathrm{m}$ up to about $1.7 \mu \mathrm{m}$, thus, giving the means to control ZDW within the third telecommunication window. 


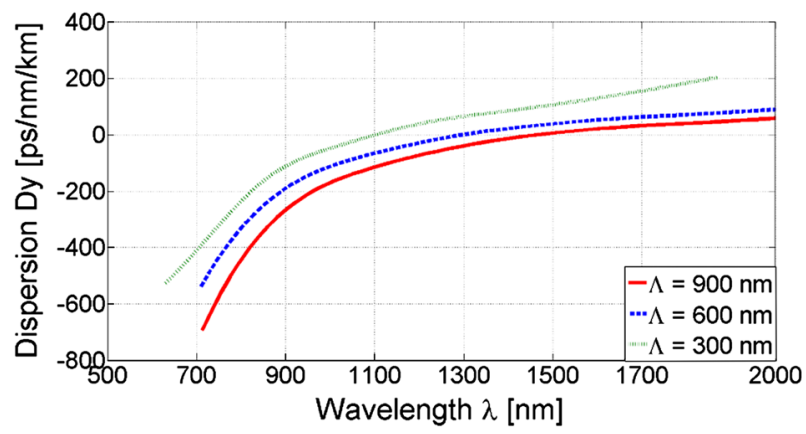

Fig. 4 Group velocity dispersion of the vertically polarized component of fundamental mode ( $E_{y}$ ) for various lattice constants $\Lambda$. The polarization components $E_{y}$ denotes direction of electric field vector perpendicular to layers in the core ( $\mathrm{Y}$ axis)

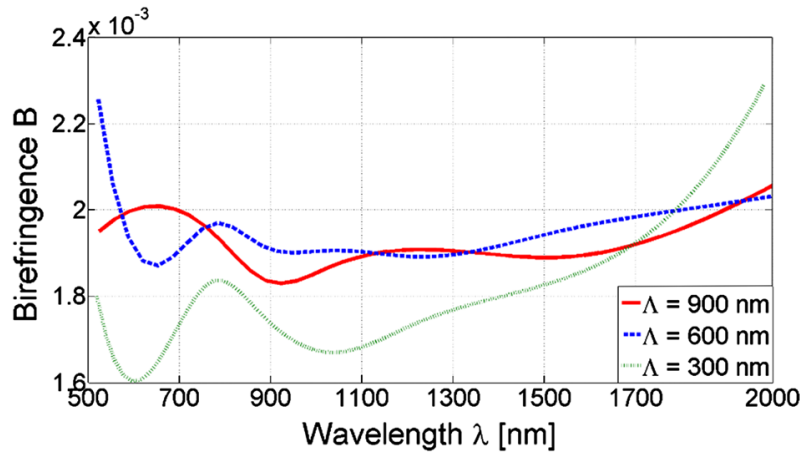

Fig. 5 The birefringence calculated for various lattice constants $\Lambda$

Table 3 Geometrical parameters of the tested fiber structures

\begin{tabular}{llllll}
\hline Lattice constant $\Lambda=300 \mathrm{~nm}$ & & & & \\
Filling factor $f$ & 0.2 & 0.5 & 1 & 2 & 5 \\
$d 1(\mathrm{~nm})$ & 50 & 100 & 150 & 200 & 250 \\
$d 2(\mathrm{~nm})$ & 250 & 200 & 150 & 100 & 50 \\
\hline
\end{tabular}

As can be seen in Fig. 5, birefringence $B$ oscillates with wavelength below $\lambda=1,000 \mathrm{~nm}$ due to resonant behavior of the core at wavelengths comparable with the lattice constant $\Lambda$. For wavelengths longer than $1,000 \mathrm{~nm}$, these oscillations are no longer observed. In particular, dispersion of birefringence remains low in the wavelength range of $1,100-1,700 \mathrm{~nm}$ for the lattice constant of $\Lambda=900 \mathrm{~nm}$, where it varies around $1.92 \times 10^{-3}$ in the range of $\pm 6 \%$ (Fig. 5).

In a subsequent step, a series of numerical simulations for different values of the filling factor for $\Lambda=300 \mathrm{~nm}$ is performed, as summarized in Table 3. Figures 6, 7 and 8 show effective refractive index, phase birefringence and dispersion of vertically and horizontally polarized components of fundamental modes, respectively.

As previously, we observe oscillations of birefringence B for wavelengths below 1,000 nm due to resonant interaction between light and anisotropic structure of the core (wavelengths shorter than 1,000 $\mathrm{nm}$ are comparable to the lattice constant $\Lambda$, Fig. 8). All considered struc- 


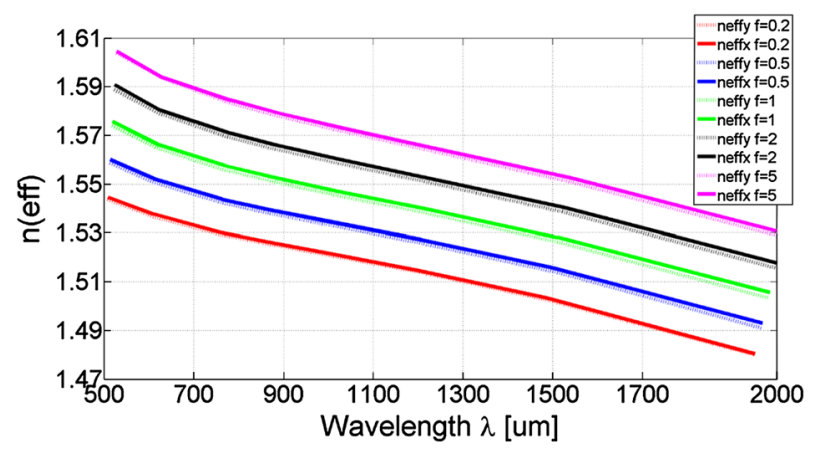

Fig. 6 Effective refractive index of vertically and horizontally polarized components of fundamental modes for the lattice constant $\Lambda=300 \mathrm{~nm}$ and various filling factors of layers in the lamellar core

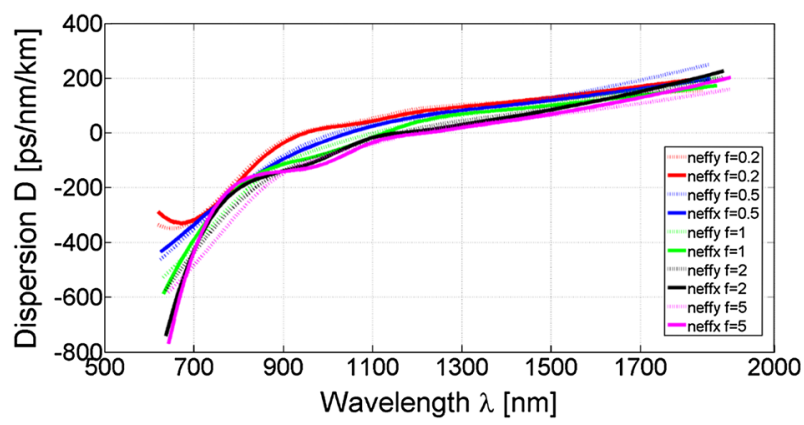

Fig. 7 Group velocity dispersion of vertically and horizontally polarized components of fundamental modes for the lattice constant $\Lambda=300 \mathrm{~nm}$ and various filling factors of layers in the lamellar core

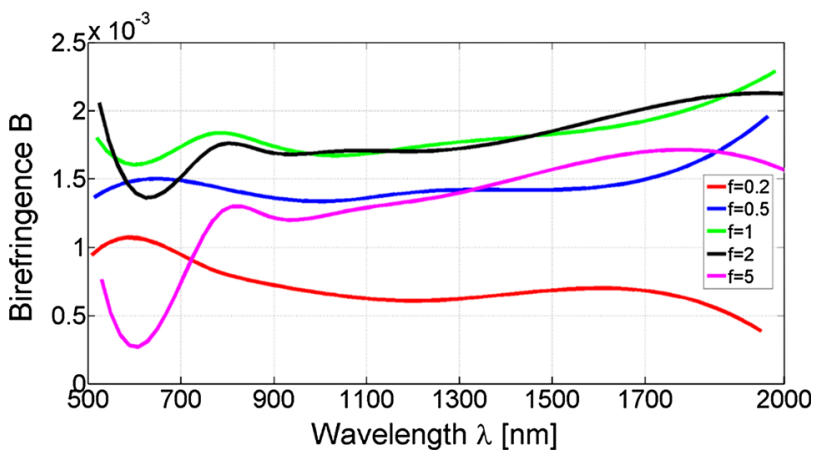

Fig. 8 Birefringence of fundamental modes for the lattice constant $\Lambda=300 \mathrm{~nm}$ and various filling factors of layers in the lamellar core

tures show low dispersion of birefringence. On the other hand, zero dispersion wavelength (ZDW) of polarization components of the fundamental mode strongly depends on filling factor. ZDW increases with the increasing $f$ from $1 \mu \mathrm{m}$ for $f=0.2$ up to about $1.3 \mu \mathrm{m}$ for $f=5$. As result, modal dispersion properties of the fiber can be adjusted with a change of filling factor, while birefringence remains almost constant. 


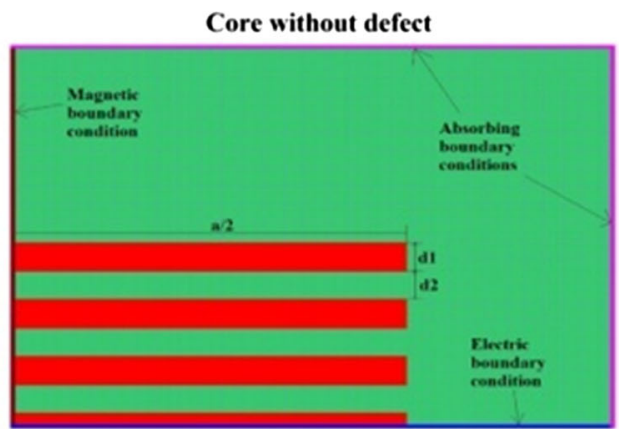

(a)

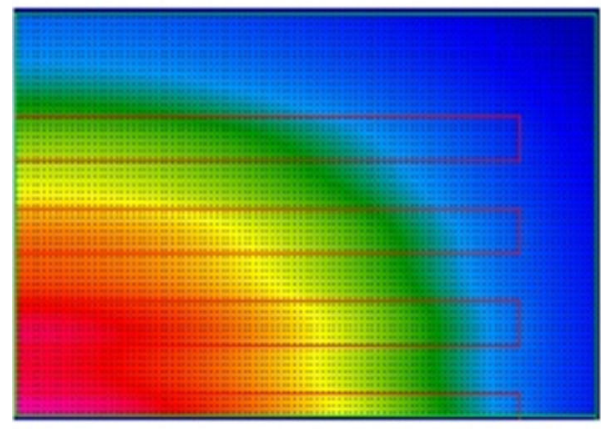

(b)

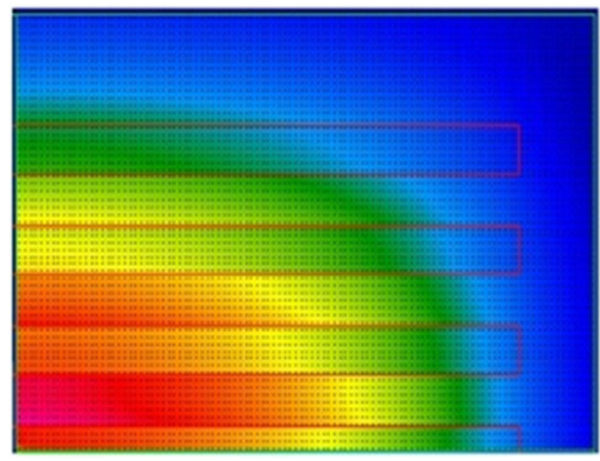

(d)

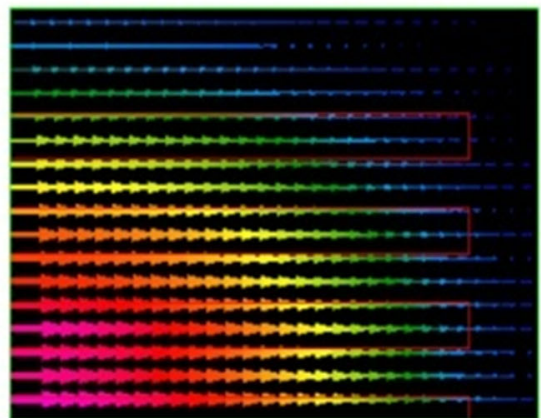

(c)

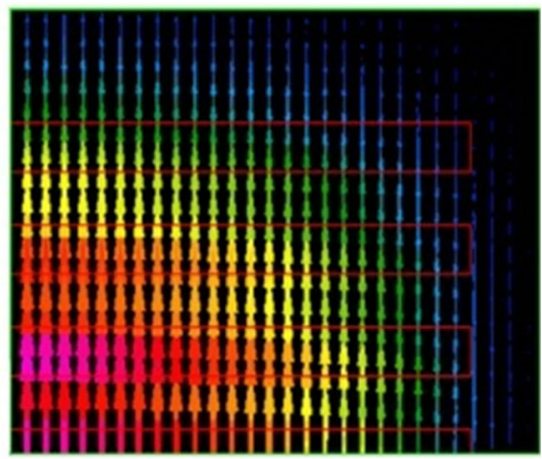

(e)

Fig. 9 a An FDTD model of a quarter of a microstructured optical fiber's cross-section without a defect; b Electric field's magnitude of a horizontally polarized component of the fundamental mode; c Electric field's vector view of a horizontally polarized component of the fundamental mode; $\mathbf{d}$ Electric field's magnitude of a vertically polarized component of the fundamental mode; e Electric field's vector view of a vertically polarized component of the fundamental mode

High values of birefringence are achieved for structures with filling factors of $f=1$ or 2. Maximum value of $2.3 \times 10^{-3}$ is obtained for wavelengths above $1.9 \mu \mathrm{m}$ when filling factor is close to 1 . In particular, dispersion of birefringence is low in the wavelength range of 900-1,700 $\mathrm{nm}$ for the structure with a lattice constant of $\Lambda=300 \mathrm{~nm}$ and filling factor of $\mathrm{f}=0.5$, where birefringence $\mathrm{B}$ varies around $1.47 \times 10^{-3}$ in the range of $\pm 6 \%$. 


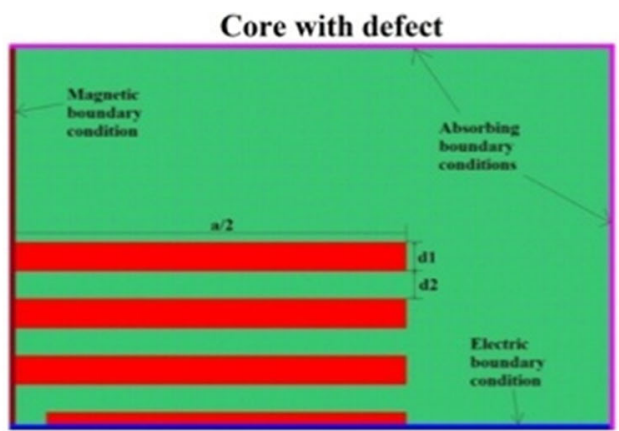

(a)

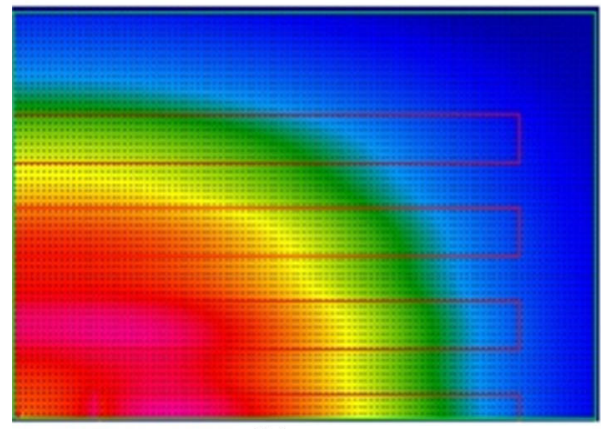

(b)

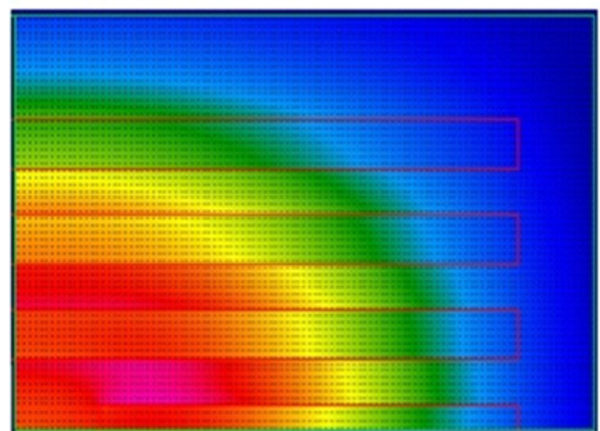

(d)

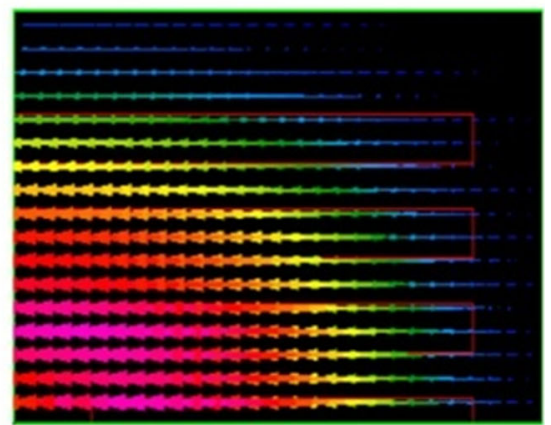

(c)

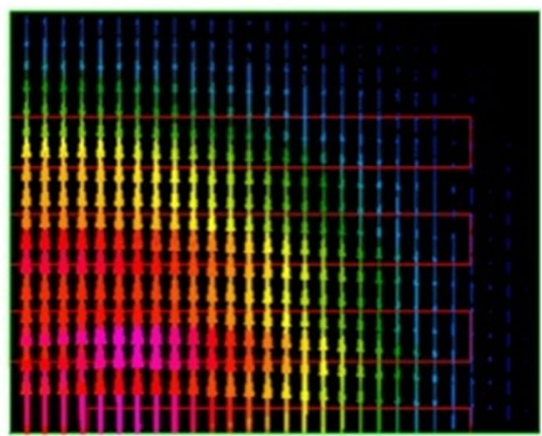

(e)

Fig. 10 a An FDTD model of a quarter of a microstructured optical fiber's cross-section with a defect; b Electric field's magnitude of a horizontally polarized component of the fundamental mode; c Electric field's vector view of a horizontally polarized component of the fundamental mode; d Electric field's magnitude of a vertically polarized component of the fundamental mode; e Electric field's vector view of a vertically polarized component of the fundamental mode

\section{Highly birefringent fiber with a subwavelength defect in the core}

To reduce further dispersion of birefringence, we consider an introduction of a subwavelength defect into the lamellar core. To verify this concept, we compared properties of the fiber with a regular lamellar core, with the fiber featuring an additional nanodefect in the core. Two scenarios are hence investigated numerically: a fiber with a nondefected core (Fig. 9) and a fiber with a subwavelength defect introduced in the core (Fig. 10). We assume a fiber 


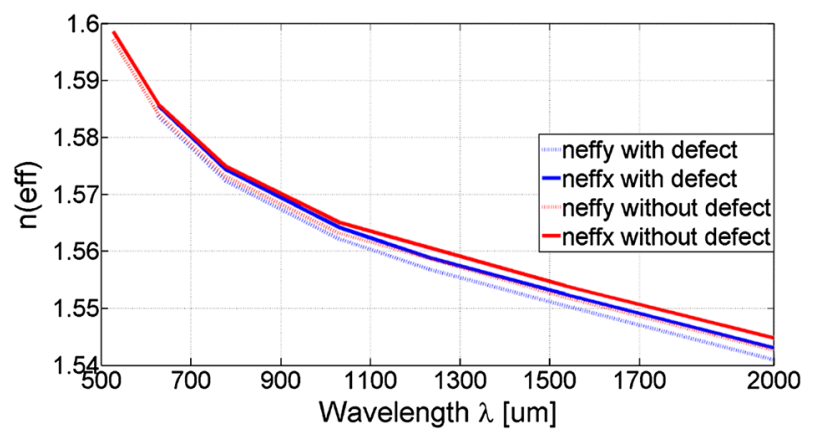

Fig. 11 The effective refractive index for both polarization components of the fundamental mode ( $E_{X}$ and $\left.\mathrm{E}_{\mathrm{y}}\right)$ in the fiber shown in Figs. 9 and 10

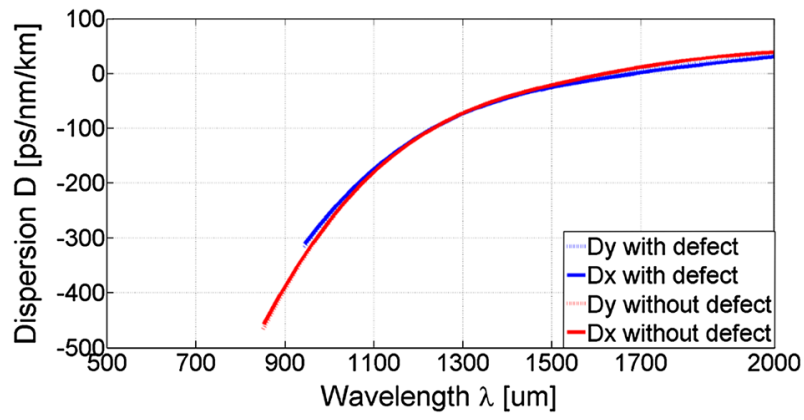

Fig. 12 Group velocity dispersion for both polarization components of the fundamental mode $\left(E_{x}\right.$ and $\left.E_{y}\right)$ in the fiber

with lamellar core with a lattice constant of $\Lambda=1,060 \mathrm{~nm}$ and filling factor $\mathrm{f}=1$. A subwavelength defect in the core is located in the middle of central layer of F2 glass. Size of defect is 2,220 nm, where F2 glass is replaced with NC21A glass (Fig. 10a).

It can be noticed in Fig. 9 that both fundamental modes are well confined inside the core. The anisotropic core does not affect the smoothness of the electric field, which is tangential to the boundaries between the host and lamellar inclusions (see Fig. 9b). On the contrary, the electric field of the vertically polarized mode is discontinuous at the aforementioned boundaries (see Fig. 9d), thus, contributing to the birefringence of those modes. However, introduction of a defect in the middle of the core decreases the magnitude of the electric field for both polarizations (see Fig. 10).

Figures 11, 12 and 13 present obtained results of numerical simulations for the structures, such as effective refractive index, dispersion and birefringence for vertical and horizontal polarization components of the fundamental mode.

The obtained results show, that for both analyzed MOF structures, high similarity of the effective refractive index and group velocity dispersion $D$ is achieved (Figs. 11, 12). On the other hand, birefringence is different for both considered cases. Dispersion of birefringence in the fiber with core including a subwavelength defect is significantly lower than in the case of a regular lamellar core. Birefringence of the fiber with a regular lamellar core is $\mathrm{B}=1.92$ and varies in the range of $600-2,000 \mathrm{~nm}$ by $\pm 15 \%$. Birefringence of the fiber with an additional subwavelength defect in the core is $\mathrm{B}=1.98$ and it varies by $\pm 5 \%$ in the range of $600-2,000 \mathrm{~nm}$ wavelengths. In particular, introduction of a defect in the core flattens the 


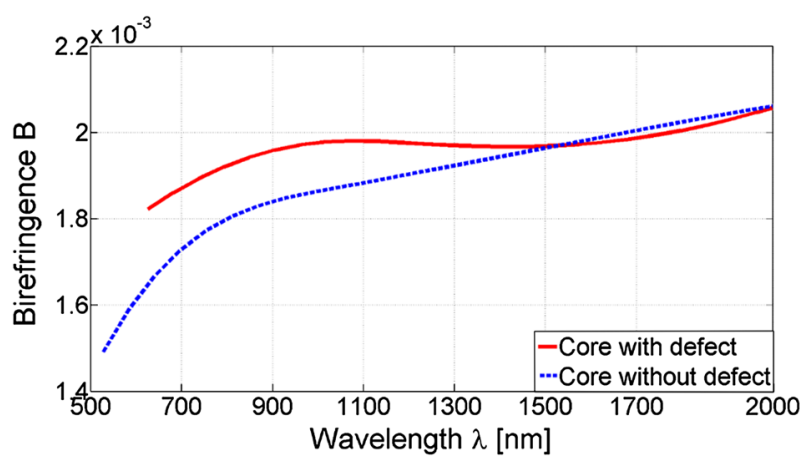

Fig. 13 Birefringence calculated for both polarization components of the fundamental mode $\left(E_{x}\right.$ and $\left.E_{y}\right)$ in the fiber shown in Figs. 9 and 10

birefringence $(\Delta B=0.03)$ in comparison to the structure without a defect $(\Delta B=0.155)$ in the rage of $900-1,650 \mathrm{~nm}$ wavelengths.

\section{Conclusions}

We have shown, that appropriate tuning of microstructure in fiber with lamellar core, enables simultaneous manipulation of the dispersion and birefringence in a certain spectral range. Silicate-based microstructured optical fibers with a core made of interlined layers of two glasses NC21 A and F2 and lattice constant of $900 \mathrm{~nm}$ enable to obtain flat birefringence over the spectral range of $600 \mathrm{~nm}(1,100-1,700 \mathrm{~nm})$ with birefringence variation below $\pm 6 \%$. Additional improvement of the structure by means of introduction of an additional defect in the lamellar core allows further reduction of birefringence dispersion. A fiber with such an additional subwavelength defect in the core enables obtaining of the birefringence at the level of $B=1.98$ with variation below $\pm 5 \%$ over the spectral range $600-2,000 \mathrm{~nm}$.

Acknowledgments This work was supported by the project TEAM/2012-9/1 operated within the Foundation for Polish Science Team Programme co-financed by the European Regional Development Fund, Operational Program Innovative Economy 2007-2013.

Open Access This article is distributed under the terms of the Creative Commons Attribution License which permits any use, distribution, and reproduction in any medium, provided the original author(s) and the source are credited.

\section{References}

Beltrán-Mejía, F., Chesini, G., Silvestre, E., George, A.K., Knight, J.C., Cordeiro, C.M.: Ultrahigh-birefringent squeezed lattice photonic crystal fiber with rotated elliptical air holes. Opt. Lett. 35, 544-546 (2010)

Buczynski, R., Kujawa, I., Pysz, D., Martynkien, T., Berghmans, F., Thienpont, H., Stepien, R.: Highly birefringent soft glass rectangular photonic crystal fibers with elliptical holes. Appl. Phys. B 99, 13-17 (2010)

Buczynski, R., Pysz, D., Stepien, R., Kasztelanic, R., Kujawa, I., Franczyk, M., Filipkowski, A., Waddie, A.J., Taghizadeh, M.R.: Dispersion management in nonlinear photonic crystal fibres with nanostructured core. J. Eur. Opt. Soc. Rapid Publ. 6, 11038 (2011)

Cerqueira Jr, S.A., Lona, D.G., de Oliveira, I., Hernandez-Figueroa, H.E., Fragnito, H.L.: Broadband singlepolarization guidance in hybrid photonic crystal fibers. Opt. Lett. 36, 133-135 (2011) 
Frazao, O., Baptista, J.M., Santos, J.L.: Recent advances in high-birefringence fiber loop mirror sensors. Sensors 7, 2970-2983 (2007)

Frazao, O., Santos, J., Araujo, Ferreira, F.L.: Optical sensing with photonic crystal fibers. Laser Photon. Rev. 2, 449-459 (2008)

Goto, R., Jackson, S.D., Fleming, S., Kuhlmey, B.T., Eggleton, B.J., Himeno, K.: Birefringent all-solid hybrid microstructured fiber. Opt. Express 16, 18752-18763 (2008)

Goto, R., Jackson, S.D., Takenaga, K.: Single-polarization operation in birefringent all-solid hybrid microstructured fiber with additional stress applying parts. Opt. Lett. 34, 3119-3121 (2009)

Klein, K.-F., Heinlein, W.E.: Orientation- and polarisation-dependent cutoff wavelengths in elliptical-core single-mode fibres. Electron. Lett. 18, 640-641 (1982)

Kujawa, I., Buczynski, R., Martynkien, T., Sadowski, M., Pysz, D., Stepien, R., Waddie, A.J., Taghizadeh, M.R.: Multiple defect core photonic crystal fiber with high birefringence induced by squeezed lattice with elliptical holes in soft glass. Opt. Fiber Technol. 18, 220-225 (2012)

Lorenc, D., Aranyosiova, M., Buczynski, R., Stepien, R., Bugar, I., Vincze, A., Velic, D.: Nonlinear refractive index of multicomponent glasses designed for fabrication of photonic crystal fibers. Appl. Phys. B: Lasers Opt. 93, 531-538 (2008)

Martynkien, T., Anuszkiewicz, A., Statkiewicz-Barabach, G., Olszewski, J., Golojuch, G., Szczurowski, M., Urbanczyk, W., Wojcik, J., Mergo, P., Makara, M., Nasilowski, T., Berghmans, F., Thienpont, H.: Birefringent photonic crystal fibers with zero polarimetric sensitivity to temperature. Appl. Phys. B 94, 635-640 (2009)

Mei, K.K., Fana, J.: Superabsorption: a method to improve absorbing boundary conditions. IEEE Trans. Antennas Propag. 40, 1001-1010 (1992)

Ortigosa-Blanch, A., Knight, J.C., Wadsworth, W.J., Arriaga, J., Mangan, B.J., Birks, T.A., Russell, P.St.J.: Highly birefringent photonic crystal fibers. Opt. Lett. 25, 1325-1327 (2000)

QuickWave-3D, QWEDSp. z o.o., 1997-2013. Available:http://www.qwed.com.pl

Salski, B., Celuch, M., Gwarek, W.: FDTD for nanoscale and optical problems. Microw. Mag. 11(2), 50-59 (2010)

Taflove, A., Hagness, S.C.: Computational Electrodynamics: The Finite-Difference Time-Domain Method. Artech House, Boston (2005)

Ulrich, R., Simon, A.: Polarization optics of twisted single-mode fibers. Appl. Opt. 18, 2241-2251 (1979)

Varnham, M.P., Payne, D.N., Birch, R.D., Tarbox, E.J.: Single-polarisation operation of highly birefringent bow-tie optical fibres. Electron. Lett. 19, 246-247 (1983)

Waddie, A.J., Buczynski, R., Hudelist, F., Nowosielski, J., Pysz, D., Stepien, R., Taghizadeh, M.R.: Form birefringence in nanostructured micro-optical devices. Opt. Mater. Express 1, 1251-1261 (2011)

Wang, A., George, A., Liu, J., Knight, J.: Highly birefringent lamellar core fiber. Opt. Express 13, 5988-5993 (2005)

Yue, Y., Kai, G., Wang, Z., Sun, T., Jin, L., Lu, Y., Zhang, C., Liu, J., Li, Y., Liu, Y., Yuan, S., Dong, X.: Highly birefringent elliptical-hole photonic crystal fiber with squeezed hexagonal lattice. Opt. Lett. 32, 469-471 (2007) 